\title{
Detailed Learning in Narrow Fields - Towards a Neural Network Model of Autism
}

\author{
Andrew P. Papliński ${ }^{1}$ and Lennart Gustafsson ${ }^{2}$ \\ 1 Computer Science and Software Engineering, \\ Monash University, Victoria 3800, Australia \\ app@csse.monash.edu.au \\ 2 Computer Science and Electrical Engineering, \\ Luleå University of Technology, S-971 87 Luleå, Sweden \\ Lennart.Gustafsson@sm.luth.se
}

\begin{abstract}
Autism is a developmental disorder in which attention shifting is known to be restricted. Using an artificial neural network model of learning we show how detailed learning in narrow fields develops when attention shifting between different sources of stimuli is restricted by familiarity preference. Our model is based on modified Self-Organizing Maps (SOM) supported by the attention shift mechanism. The novelty seeking and the attention shifting restricted by familiarity preference learning modes are investigated for stimuli of low and high dimensionality which requires different techniques to visualise feature maps. To make learning more biologically plausible we project the stimuli onto a unity hyper-sphere. The distance between a stimulus and a weight vector can now be simply measured by the post-synaptic activities. The modified "dot-product" learning law that keeps evolving weights on the surface of the hyper-sphere has been employed.
\end{abstract}

\section{Introduction: Autism, Restricted Attention Shifting}

Autism is a developmental disorder with diagnostic criteria (DSM-IV, 1994 [1]) grouped in three categories: impairments in social interaction, impairments in verbal and nonverbal communication, and restricted repetitive and stereotyped patterns of behavior, interests, and activities. The subcategory of the last category: "encompassing preoccupation with one or more stereotyped and restricted patterns of interest that is abnormal either in intensity or focus" is of particular interest in this paper. It is generally agreed that attention shifting is not normal in autism but the underlying cause is a matter of debate with two main hypotheses, a general attention shifting impairment [2], and attention shifting restricted by familiarity preference or novelty avoidance 3456. A brief review of these hypotheses can be found in [7].

In this paper an artificial neural network model of learning is used to show how detailed learning in narrow fields develops when attention shifting between different sources of stimuli is restricted by familiarity preference. This is a continuation of our earlier work on modelling autism presented in [78] where we examined the use of Kohonen Self-Organizing Maps subjected to two-dimensional 
data. We found that attention shifting restricted by familiarity preference causes the feature map to learn the data from the source with the least variability in its data whereas normal learning, i.e. learning with attention shifts to that source which presents new data, resulted in maps which adapt to the data from both sources. Learning under general attention shifting impairment resulted in maps that were very similar to the maps obtained from normal learning.

\section{Artificial Neural Networks and Learning in Autism}

Our model of autistic learning is based on Kohonen Self-Organizing Maps (SOMs) 9] with some modifications and addition of the attention shift mechanism. It is well known that a Self-Organizing Map is a competitive neural network in which $m$ neurons, each with $p$ synapses are organized in an $l$-dimensional lattice (grid) representing the feature space. Such a neural network performs mapping of a $p$-dimensional input space into the $l$-dimensional feature space. In Figure 1 we present an example of a self-organizing map consisting of $m=12$

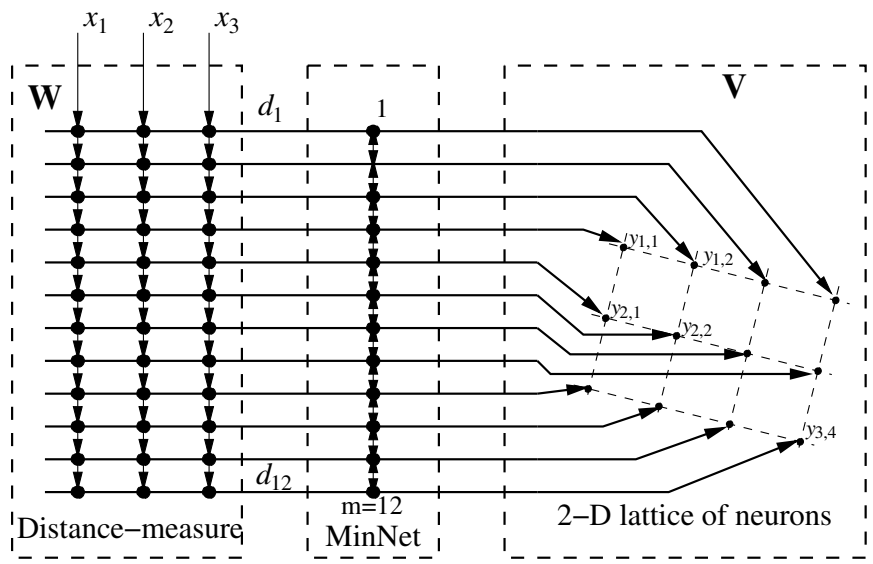

Fig. 1. A 2-D SOM with $p=3 ; m=\left[\begin{array}{ll}3 & 4\end{array}\right] ; l=2$

neurons in which the input space is 3-dimensional $(p=3)$ and the feature space is 2-dimensional $(l=2)$. The first section of the network is a distancemeasure layer consisting of $m=12$ dendrites each containing $p=3$ synapses excited by $p$-dimensional stimuli $\mathbf{x}$ and characterised by the $p$-dimensional weight vector $\mathbf{w}_{i}, i=1, \ldots, m$. The distance-measure layer calculates the distances $d_{i}$ between each input vector $\mathbf{x}$ and every weight vector $\mathbf{w}_{i}$. This distance vector, $\left.\mathbf{d}=\left[d_{1}, \ldots, d_{m}\right)\right]$ is passed to the competition layer, the MinNet in Figure [1 which calculates the minimal distance $d_{k}=\min d_{i}$ in order to establish the position of the winning neuron $k$. The competition is implemented through the lateral inhibitive and local self-excitatory connections between neurons in the competitive layer. In addition, every neuron is located at $l=2-\mathrm{D}$ lattice and its 
position is specified by an $l$-dimensional vector $\mathbf{v}_{i}$. The synaptic weight vectors, $\mathbf{w}_{i}$, and the vectors of topological positions of neurons, $\mathbf{v}_{i}$, are grouped into the $m \times p$ weight matrix $W$ and $m \times l$ position matrix $V$.

In order to make the distance calculations simpler and more biologically plausible we project our $(p-1)$-dimensional stimuli $\hat{\mathbf{x}}(n)$ onto a unity $p$-dimensional sphere.

$$
\mathbf{d}=W \cdot \mathbf{x}=\cos \boldsymbol{\alpha}, \quad d_{j} \in[-1,+1]
$$

equal to the cosine of the angles between the stimulus and the weight vectors.

For the normalised stimuli, in order to keep the weight vectors on the surface of the unity sphere we used the "dot-product" learning law 9]. For the $j$ th neuron we can write:

$$
\hat{\mathbf{w}}_{j}=\mathbf{w}_{j}+\eta \cdot \Lambda_{j} \cdot\left(\mathbf{x}^{T}-\mathbf{w}_{j}\right), \quad \mathbf{w}_{j}(n)=\frac{\hat{\mathbf{w}}_{j}}{\left\|\hat{\mathbf{w}}_{j}\right\|}
$$

where $\mathbf{x}$ is the current stimulus, $\mathbf{w}_{j}, \mathbf{w}_{j}(n)$ are the current and the next weight vectors for the $j$ th neuron, respectively, $\Lambda_{j}$ is the value of the neighbourhood function, and $\eta$ is the learning gain. Normalization as in eqn (2) is computationally relatively complex, but for small learning gain $\eta<1$ it can be shown that the weight update can be expressed in the following elegant form:

$$
\mathbf{w}_{j}(n)=\mathbf{w}_{j}+\eta \cdot \Lambda_{j} \cdot\left(\mathbf{x}^{T}-\mathbf{w}_{j} \cdot d_{j}\right), \quad d_{j}=\mathbf{w}_{j} \cdot \mathbf{x}
$$

The modification that pushes the updated weights towards the unity sphere is based on introduction of the post-synaptic activity, $d_{j}$, into the update equation.

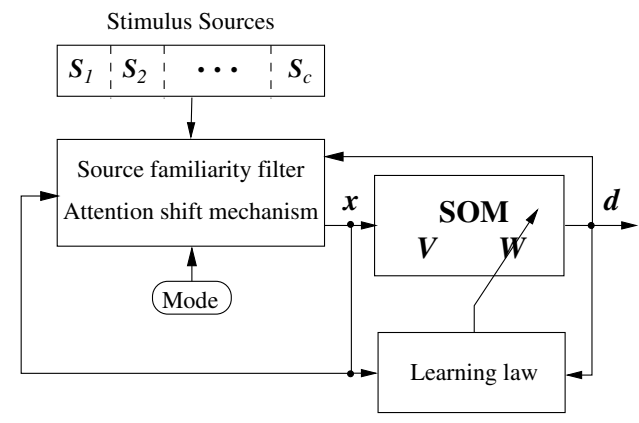

Fig. 2. A block-diagram of the model of autistic learning

The block-diagram of the model of autistic learning which includes source familiarity filter and attention shift mechanism is presented in Figure 2. The central part is the SOM neural network as presented in Figure 1 together with the learning section implementing the learning law, or map formation algorithm, given in eqn (3). At each learning step a stimulus is randomly generated from 
one of the sources, $S_{1}, \ldots S_{c}$. The attention shifting mechanism determines if that stimulus is presented to the map for learning.

In the normal, or novelty seeking learning mode, attention is shifted to another source if the new stimulus originates from that source.

In the attention shifting restricted by familiarity preference learning mode attention is shifted to another source if that source presents the next new stimulus, but conditionally, depending on the map's familiarity with that source. The map familiarity to a particular source is measured by the time averaged value of the distance between map nodes and the stimuli. When both sources are unfamiliar to the map, i.e. in the beginning stage of self-organization, attention is shifted to an alternate source if that source presented the next stimulus as in the novelty seeking mode. As the map develops some familiarity with the sources, i.e. the node weights begin to resemble the data, attention is shifted with a higher probability to the source which is most familiar to the map. If the map becomes familiar to two or more sources (the average difference between node weights and the data from the sources becomes smaller than a predetermined small value) then attention is unconditionally shifted.

\section{Modelling Autistic Learning with Low-Dimensional Stimuli}

In the first set of simulations we use three sources generating four classes of two-dimensional stimuli, each class consisting of just two exemplars. Therefore we have in total twenty four two-dimensional stimuli. For the sake of conceptualization we can imagine that the the sources produce three dialects of a very limited protolanguage, each with four protophonemes (classes) pronounced in two slightly different ways. The neural network which is used to model the stimuli consists of sixteen neurons (or nodes) organized in a $4 \times 4$ two-dimensional lattice, or grid.

Thanks to the low-dimensionality of stimuli (and weights) it is possible to visualise the feature map in the input space. Such a feature map is a plot of synaptic weights in the input space in which weights of the neighbouring neurons are joined by lines and illustrates the mapping from two-dimensional input space to two-dimensional feature space as shown in Figure 3a. The stimuli originating from a particular source are marked with '+', 'o' and ' $\star$ ', respectively, and are arranged in four pairs (classes). The neuronal nodes are marked with ' $*$ ', and joined by the straight lines. The map shows the way in which the neuronal $4 \times 4$ lattice approximates the input data (stimuli).

The map of Figure 3 a has been developed in the novelty seeking mode, that is, in normal learning. Normal learning results in a map where most neurons are "shared" between two stimuli and thereby all stimuli have a good representation in the map. There are also a few "dead" neurons in the map, neurons that are far from all stimuli; this is a very common thing to occur.

There are two parameters visible on the map, namely the attention shift index, and the map "goodness" index. The attention shift index is the num- 

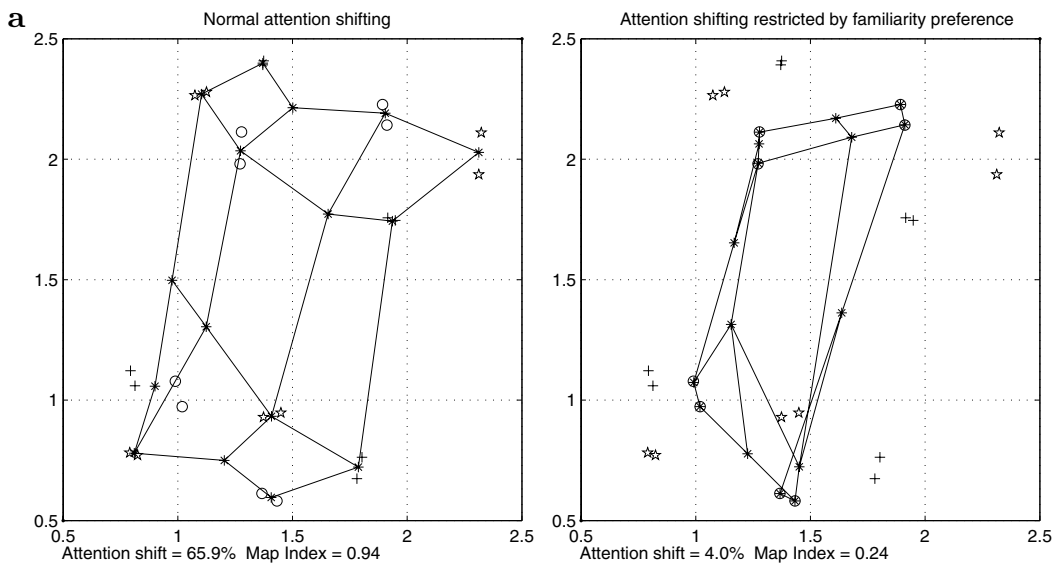

Fig. 3. A $4 \times 4$ feature map in the 2 -D input space developed in the: a. novelty seeking, and $\mathbf{b}$. attention shifting restricted by familiarity preference learning modes

ber of attention shifts normalised with the total number of stimuli used during learning. With three sources and equal probability of generating a stimulus from each source the attention shift index is clearly equal to $2 / 3$. The map "goodness" index is formed from the sum of distances between the class means and weights located in the proximity of the means. This sum of distances will be the largest for the initial value of weights and will be reduced during training. The "goodness index" has been normalized so that a value of 1 represents a map with nodes located at the centres of all data subclasses.

After all these introductions we are ready to consider detailed learning in a narrow field characteristic to autistic learning. The feature map presented in Figure 3b is the result of learning when the attention shifting is restricted by familiarity preference. In this mode of learning the attention shifting has ceased (attention shift index is $4 \%$ ) and as a result only the source with the lowest variability (spread), in this case marked by 'o', is learned. The representational capacity of the map now makes it possible to assign one neuron to each of the eight stimuli, i.e. detailed learning in a narrow field, characteristic to autistic learning, has occurred.

\section{Modelling Autistic Learning with High-Dimensionality Stimuli}

In the second set of simulations the width of the afferent connections, that is, the dimensionality of the stimuli and weight vectors is much higher $(p=18)$. As previously, the neural network which is used to model the stimuli consists of sixteen neurons organized in a $4 \times 4$ two-dimensional grid. With such a high dimensionality the feature map cannot now be visualised in the input space. However the dimensionality of the feature space, that is, the neuronal grid is 
still low $(l=2)$ and the feature maps can be illustrated in this space by attaching to each neuron stimuli located in the proximity of the relevant weight vector.

The higher-dimensional stimuli are here chosen to be animals and a number of their characteristics, mostly visual. One source contains animals of widely different kinds: mammals, birds, a reptile and fish. The other source contains only cats. There are animals which stand out and there are other animals that are very similar to each other, in some cases separated only by coloration. The animals are listed below:

Source A: Przewalski's horse, Grevy's zebra, Canis lupus (wolf), Dingo, White (mute) swan, Black swan, Atlantic salmon, Rainbow trout, Polar bear, Kodiak bear, White rhinocerous, Hippopotamus, Grey Western kangaroo, Swamp wallaby, Anaconda, Grey whale

Source B: even colored domestic cat, striped domestic cat, black panther, leopard, ocelot, jaguar, lion tiger.

For investigating the generalization properties acquired by the map during learning we also have four test animals: black domestic cat, Siamese cat, snow leopard and Eurasian lynx. The animals have been characterized by weight, food, locomotion (fins, wings, two legs, four legs), feet (hooves, claws or other), coloration (black, white, even colored, spotted, striped), facial feature (elongated or short nose), aquatic preference and social structure. Each animal is described by eighteen numbers. We project the animal characterization on a 19-dimensional unity sphere as described in Sect. 2.

In order to assess the categorization characteristics of the maps it is necessary to establish the "likeness" among these animals. In Table 1 pairs of greatest likeness are presented and a measure of their angular distance on the hypersphere. The first animal is chosen and then the animal that most resembles it is calculated. It is clear from Table 1 that the anaconda and the whale stand out

Table 1. Angular distances in the pairs of the closest animals

\begin{tabular}{|llr|}
\hline \multicolumn{3}{|c|}{ Source A } \\
\hline \hline Horse & Zebra & 28 \\
Zebra & Horse & 28 \\
Wolf & Dingo & 55 \\
Dingo & Wolf & 55 \\
WSwan & Bswan & 38 \\
BSwan & WSwan & 38 \\
Salmon & Trout & 36 \\
Trout & Salmon & 36 \\
PBear & KBear & 50 \\
KBear & Pbear & 50 \\
Rhino & Hippo & 42 \\
Hippo & Rhino & 42 \\
Kangaroo & Wallaby & 44 \\
Wallaby & Kangaroo & 44 \\
Anaconda & PBear & 103 \\
Whale & Hippo & 406 \\
\hline
\end{tabular}

\begin{tabular}{|llr|}
\hline \multicolumn{3}{|c|}{ Source B } \\
\hline \hline CatEcld & catSiam & 9 \\
catstrp & catSiam & 9 \\
panther & leopard & 12 \\
leopard & panther & 12 \\
ocelot & lynxEur & 41 \\
jaguar & tiger & 40 \\
lion & tiger & 45 \\
tiger & jaguar & 40 \\
\hline \hline \multicolumn{3}{|c|}{ Test cats } \\
\hline \hline catBlck & catSiam & 9 \\
catSiam & catEcld & 9 \\
snowLprd leopard & 13 \\
lynxEur & ocelot & 41 \\
\hline
\end{tabular}


and that the domestic cats are very similar to each other. There are also many pairs like horse - zebra and a group of three similar big cats, jaguar, lion and tiger. A good map of only sixteen nodes would thus assign one node each for the whale and the anaconda, let the domestic cats share one node, let the three big cats share one node and let a number of pairs of animals share one node per pair. In a map formed by attending to Source B only, a good map would assign one node to each of the cats.

The feature map resulting from the normal, novelty seeking learning is shown in Figure 4 a. The map consists of the $4 \times 4$ neuronal grid. Each animal is shown

a

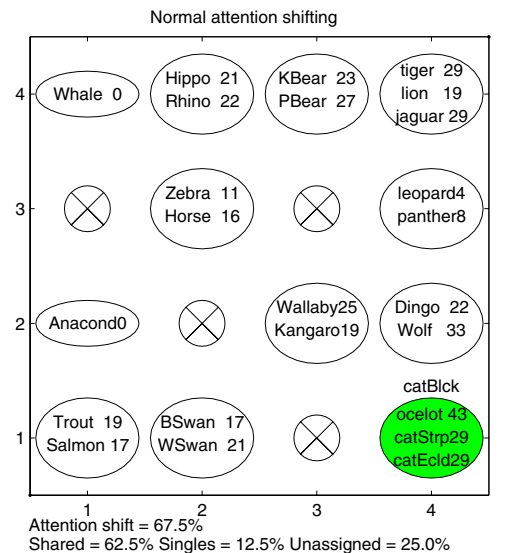

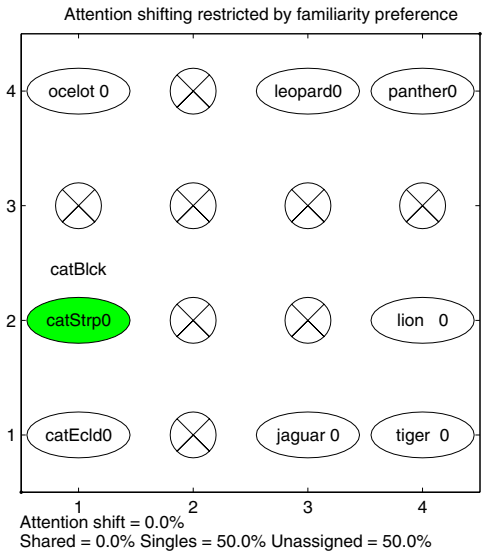

Fig. 4. The feature maps developed in the a. novelty seeking, and b. attention shifting restricted by familiarity preference learning modes. The shaded ovals represent the network response to a test animal

at the node with the best match of weights. The angular distance between the animal vector and the node weight vector is shown after the animal name. In the map the number of animals for which a given node is the closest varies from three to zero ("dead neurons"). As expected the whale is represented by its own node with distance 0 , i.e. a perfect match between the animal and the best matching node has been achieved. As also anticipated the three big cats share one node almost in the middle between them. Other animals are likewise represented as anticipated. Four nodes are dead (unassigned).

The feature map resulting from learning in the attention shifting restricted by familiarity preference mode is shown in Figure 4b. This map, which developed when the attention shifting has ceased, reveals the characteristics of detailed learning in a narrow field, that is, only the stimuli source with the lowest variability, i.e. the cats, is learned. Every cat is represented by its best matching node and all these best matches are perfect because the number of neurons considerably exceeds the number of cats. Several of the nodes marked as "unassigned" 
have weight vectors which are close to the cats which have been allocated to neighboring (closest match) neurons.

Testing for Generalization: A good map should have acquired some generalization capability, i.e. when presented with a stimulus it has not learned, it should be represented by a node with weights that are similar if such a node exists. We presented the maps from Figures $4 \mathrm{a}$ and $4 \mathrm{~b}$ with the "Black Cat" stimulus which has not been used in learning. In both cases the node closest to this test stimulus is the one allocated to the striped cat, possibly shared with other similar cats. These nodes are shaded in Figure 4.

\section{Conclusion}

Self-organization of feature maps presented with stimuli from more than one source will result in very different maps depending on the rules for attention shifting between the sources. In learning under novelty seeking the resulting map will represent all sources and group stimuli in an "economic" way — similar stimuli will share the same node. In learning under familiarity preference - a characteristic well-known in autism - the resulting map will represent only the map with the lowest variability and will use its representational capacity so that stimuli with very minor differences, such as the coloration of domestic cats, each have their own nodes. The learning which is modelled by such self-organization corresponds to detailed learning in narrow fields, a characteristic well-known in autism.

Acknowledgements. This work is a result of a cooperation between Luleå University of Technology, Luleå, Sweden and Monash University, Clayton, Australia. We wish to express our appreciation to the Swedish STINT grant scheme and the Monash School of Computer Science and Software Engineering for supporting this cooperation.

\section{References}

1. Diagnostic and statistical manual of mental disorders. 4th ed. American Psychiatric Association (1994) Available from: http://www.psychologynet.org.

2. Courchesne, E., Townsend, J., Akshoomoff, N., Saitoh, O., Yeung-Courchesne, R., Lincoln, A., James, H., Haas, R., Schreibman, L., Lau, L.: Impairment in shifting attention in autistic and cerebellar patients. Beh. Neuroscience 108 (1994a) 848-865

3. Kootz, J., Marinelli, B., Cohen, D.: Modulation of response to environmental stimulation in autistic children. Jour. Autism Dev. Dis. 12 (1982) 185-193

4. Dawson, G., Meltzoff, A., Osterling, J., Rinaldi, J., Brown, E.: Children with autism fail to orient to naturally occurring social stimuli. Jour. Autism Dev. Dis. 28 (1998) 479-485

5. Pascualvaca, D., Fantie, B., Papageorgiou, M., Mirsky, A.: Attentional capacities in children with autism: Is there a general deficit in shifting focus? Jour. Autism Dev. Dis. 28 (1998) 467-478 
6. Minshew, N., Luna, B., Sweeney, J.: Oculomotor evidence for neocortical systems but not cerebellar dysfunction in autism. Neurology (1999) 917-922

7. Gustafsson, L., Papliński, A.P.: Self-organization of an artificial neural network subjected to attention shift impairments and novelty avoidance: Implications for the development of autism. Jour. Autism Dev. Dis. (2002) to be published.

8. Papliński, A.P., Gustafsson, L.: An attempt in modelling autism using selforganizing maps. In: Proc. 9th Int. Conf. Neural Inf. Proc., Singapore (2002) 301304

9. Kohonen, T.: Self-Organising Maps. 3rd edn. Springer-Verlag, Berlin (2001) 